\title{
Band bending driven evolution of the bound electron states at the interface between a three-dimensional topological insulator and a three-dimensional normal insulator
}

\author{
V. N. Men'shov, ${ }^{1,2,3}$ V. V. Tugushev,,${ }^{1,2,3,4}$ S. V. Eremeev, ${ }^{2,5,6}$ P. M. Echenique, ${ }^{3,7}$ and E. V. Chulkov ${ }^{2,3,6,7}$ \\ ${ }^{1}$ NRC Kurchatov Institute, Kurchatov Sqr. 1, 123182 Moscow, Russia \\ ${ }^{2}$ Tomsk State University, 634050 Tomsk, Russia \\ ${ }^{3}$ Donostia International Physics Center (DIPC), 20018 San Sebastián/Donostia, Basque Country, Spain \\ ${ }^{4}$ A.M. Prokhorov General Physics Institute, Vavilov str. 38, 119991 Moscow, Russia \\ ${ }^{5}$ Institute of Strength Physics and Materials Science, 634021, Tomsk, Russia \\ ${ }^{6}$ Saint Petersburg State University, Saint Petersburg, 198504, Russia \\ ${ }^{7}$ Departamento de Física de Materiales UPV/EHU, Centro de Física de Materiales CFM - MPC \\ and Centro Mixto CSIC-UPV/EHU, 20080 San Sebastián/Donostia, Basque Country, Spain \\ (Received 30 October 2014; revised manuscript received 25 January 2015; published 17 February 2015)
}

\begin{abstract}
In the frame of $\mathbf{k} \cdot \mathbf{p}$ method and variational approach for the effective energy functional of a contact between a three-dimensional topological insulator (TI) and normal insulator (NI), we analytically describe the formation of interfacial bound electron states of two types (ordinary and topological) having different spatial distributions and energy spectra. We show that these states appear as a result of the interplay of two factors: hybridization and band bending of the TI and NI electron states near the TI/NI boundary. These results are corroborated by the density functional theory calculations for the exemplar $\mathrm{Bi}_{2} \mathrm{Se}_{3} / \mathrm{ZnSe}$ system.
\end{abstract}

DOI: 10.1103/PhysRevB.91.075307

PACS number(s): 74.25.Jb, 73.20.-r

\section{INTRODUCTION}

The physical origin, peculiarities and methods of description of bound electron states at the interface formed by a three-dimensional (3D) topological insulator (TI) and normal insulator (NI) are the subject of considerable interest (see, for example, Refs. [1-3] and references therein). Such the interest is due to predicted unusual properties of the TI/NI heterostructures, but to utilize them in next-generation nanoelectronic devices, one should take an important step to drive the characteristics of interfacial electron states in the design of the TI/NI systems. In turn, these characteristics result from a "twofold" nature of bound electron states at the TI/NI contact.

On the one hand, the appearance of the bound electron states at the TI/NI contact is due to quantum proximity effect caused by an effective interfacial potential at the boundary between two insulators (semiconductors) with different crystal symmetries, lattice parameters, band gaps, and electron affinities. This potential arises from the one-electron hybridization of the TI and NI electron states, as well as redistribution of the charge and spin densities on the both sides of the interface. Hence, it defines the boundary conditions for the electron wave functions (envelope functions in the $\mathbf{k} \cdot \mathbf{p}$ method) at the interface, the form of the electron spectrum, spin texture, and spatial localization of interfacial electron states [4]. But for all that, both the strong spin-orbit coupling (SOC) and the inverted gap of the spectrum, which are essential features of the electron structure of the bulk TI, can play a significant although not decisive role.

On the other hand, the existence of helical Dirac-like interfacial electron states in TI/NI heterostructures is formally provided by the bulk-boundary correspondence theorem formulated in the topological band theory of solids [5,6]. For a layered system containing alternating slabs of TI and NI materials with different topological invariants, this theorem predicts an appearance of helical bound electron states inside the energy gap in the electron spectrum. So, the "quantum-mechanical" arguments based on the quantum proximity effect driven by an effective interfacial potential, as well as "topological" arguments based on the bulk-boundary correspondence paradigm, predict an existence of interfacial bound electron states in the TI/NI heterostructures.

Numerical calculations based on density functional theory (DFT) methods have shown a very unusual picture of the space and energy relocation of interfacial electron states in some TI/NI heterostructures composed of the TI and NI slabs $[7,8]$. The principal effect in these systems is the formation of interfacial bound electron states of two different types. Recently, in Ref. [9], this phenomenon has been preliminarily studied with an analytical approach. Within a quasiparticle concept it was demonstrated that the spectrum of interfacial bound electron states contains "ordinary" and "topological" branches with different characteristic lengths, probability maxima, and peaks of spectral weight in the energy scale. The analytical description in Ref. [9] was based on the simplified model of an effective short-range interface (pseudo)potential provided by hybridization of the TI and NI electron states at the TI/NI interface. It was predicted that, even within this model, interfacial bound electron states exist near the TI/NI boundary. However, in many real TI/NI heterostructures the TI and NI components are, respectively, narrow-gap and wide-gap semiconductors with different electron affinities. It is evident that, in such systems, significant electrostatic (Coulomb) potential is induced due to strong charge-density redistribution between TI and NI materials near their contact. Since this potential, which has been not considered until now, contains the components of different spatial scales, it may lead to a significant band-bending effect, which is particularly important on the TI (narrow-gap) side of the contact.

Generally speaking, the TI boundary states have a rather wide range of tunability of the electronic properties under various perturbations, such as structural distortions $[10,11]$ and mechanical strains [12,13], adsorption of (magnetic and 
nonmagnetic) atoms and molecules on the surface [14-17], an alternation of the surface termination $[18,19]$, engineering via capping layers and interfaces with other materials [20-23], applying an external gate voltage [20,24,25], etc., some of which are accompanied by electrostatic bending of actual bands in TIs. We only briefly annotate the interesting consequences of these perturbations for the states confined at the TI boundary (of course, our literature survey is not exhaustive). For instance, angle-resolved photoemission spectroscopy (ARPES) measurements of the prototypical 3D TIs $\mathrm{Bi}_{2} \mathrm{Se}_{3}$ and $\mathrm{Bi}_{2} \mathrm{Te}_{3}$ have, upon exposure to various environments, shown topological-order robustness [26]. Nevertheless, the surface states were strongly modified due to an exposure to $\mathrm{N}_{2}$ or air, moreover, the formation of two-dimensional (2D) quantum well states near the exposed TI surface have been observed. The spectral changes at the $\mathrm{Bi}_{2} \mathrm{Se}_{3}(111)$ surface similar to those reported in Ref. [26] were demonstrated in connection with the adsorption of carbon monoxide $\mathrm{CO}$ or $\mathrm{Fe}$ in Refs. [14,15], respectively. The Rashba-split quantum well states of the conduction band were observed and interpreted as additional surface states. In Refs. [27,28], in density functional calculations (DFT), it was found that the $\mathrm{K}$ adatoms on ultrathin films of $\mathrm{Bi}_{2} \mathrm{Se}_{3}$ induce downward band bending within 2 to $3 \mathrm{~nm}$ from the surface, due to charge transfer from the adatoms to the TI, which lead to a Dirac-cone state, localized slightly deep into the TI. There are now intensive experimental and theoretical efforts on studying the interface states in various 3D TI-based heterostructures that carry the signature of both topology and contact conditions [1$3,20-23,29,30]$. Below we throw light on the role of the band-bending effect in the formation of the bound electron states at the TI/NI interface.

The paper is organized as follows: In Sec. II, we briefly discuss the genesis of bound electron states within a variational approach for the energy functional based on the concept of effective interfacial potential at the TI/NI contact. In Sec. III, we canvass evolution of interfacial bound electron states under the band-bending effect. In Sec. IV, we outline the results of $a b$ initio DFT calculations for the $\mathrm{Bi}_{2} \mathrm{Se}_{3} / \mathrm{ZnSe}$ system. In Sec. V, we compare the numerical and analytical results, establish a correspondence between parameters used in both approaches, and thus interpret the DFT calculation results in terms of the proposed analytical approach. In Sec. VI, we summarize the results obtained.

\section{GENESIS OF INTERFACIAL BOUND ELECTRON STATES IN A VARIATIONAL APPROACH}

First, let us make a brief reminder on an analytical description of the bound electron states at the 3D TI/NI interface which was suggested in Refs. [9,31]. The relevant theoretical model is based on the four-band $\mathbf{k} \cdot \mathbf{p}$ Hamiltonian with strong SOC proposed in Ref. [32] to describe the low-energy and long-wavelength bulk electron states near the $\Gamma$ point of the Brillouin zone in the narrow-gap semiconductor $\mathrm{Bi}_{2} \mathrm{Se}_{3}$ as a prototypical 3D TI. We make use the simplified isotropic version of the Hamiltonian in the form

$$
\mathbb{H}_{T}(\mathbf{k})=\Xi(\mathbf{k}) \tau_{z} \otimes \sigma_{0}+\mathrm{A} \tau_{x} \otimes(\mathbf{k} \cdot \boldsymbol{\sigma})
$$

where $\Xi(\mathbf{k})=\Xi-\mathrm{B} k^{2}, \mathbf{k}$ is the wave vector, $k=|\mathbf{k}|$, and $\sigma_{\alpha}$ and $\tau_{\alpha}(\alpha=0, x, y, z)$ denote the Pauli matrices in the spin and orbital space, respectively. The Hamiltonian (1) is written in the basis $u=\{|+, \uparrow\rangle,|-, \uparrow\rangle,|+, \downarrow\rangle,|-, \downarrow\rangle\}$ of the four states at the $\Gamma$ point with $\mathbf{k}=0$. The superscripts \pm denote the even- and odd-parity states and the arrows $\uparrow, \downarrow$ indicate the spin projections onto the $z$ quantization axis. In the $\mathrm{Bi}_{2} \mathrm{Se}_{3}$-type materials, these four states originate from the bonding combinations of $p_{z}$ orbitals of bismuth and antibonding combinations of $p_{z}$ orbitals of selenium [32]. The parameters $\Xi, \mathrm{B}$, and $\mathrm{A}$ are connected with matrix elements of momentum [32]. The simple model in Eq. (1) captures the remarkable feature of the band structure consisting in the inverted order of the energy terms around the $\Gamma$ point (as compared with the large $\mathbf{k}$ ) under the condition $\Xi, \mathrm{B}>$ 0 , which correctly characterizes the topologically nontrivial nature of the TI side of the system due to strong SOC.

One assumes that constituents of the TI/NI heterostructure are joined at a perfectly flat interface located at $z=0$ so that 3D TI occupies the right half space, $z>0$, while the NI occupies the left one, $z<0$. The full electron energy of the $\mathrm{TI} / \mathrm{NI}$ heterostructure is

$$
\begin{gathered}
\Omega=\Omega_{T}+\Omega_{N}+\Omega_{I} \\
\Omega_{T}=\int_{z>0} d \mathbf{r} \Theta^{\dagger}(\mathbf{r})\left[\mathbb{H}_{T}(-i \nabla)+\varphi(\mathbf{r}) \mathbb{I}\right] \Theta(\mathbf{r}) \\
\Omega_{N}=\int_{z<0} d \mathbf{r} \Phi_{N}^{\dagger}(\mathbf{r}) \mathbb{H}_{N}(-i \nabla) \Phi_{N}(\mathbf{r}), \\
\Omega_{I}=\int d \mathbf{r}\left[\Theta^{\dagger}(\mathbf{r}) \mathbb{V}_{N}(\mathbf{r}) \Phi_{N}(\mathbf{r})+\Phi_{N}^{\dagger}(\mathbf{r}) \mathbb{V}_{N}^{\dagger}(\mathbf{r}) \Theta(\mathbf{r})\right]
\end{gathered}
$$

On the TI side, in Eq. (3), the operator $\mathbb{H}_{T}(-i \nabla)$ (momentum $\mathbf{k}$ is replaced by operator $-i \nabla$ ) determined in Eq. (1) acts in the space of the spinor envelope functions $\Theta(\mathbf{r})=$ $\left(\theta_{1}(\mathbf{r}), \theta_{2}(\mathbf{r}), \theta_{3}(\mathbf{r}), \theta_{4}(\mathbf{r})\right)^{\operatorname{tr}}$ that are represented in the basis $u$. In Eq. (4) the Hamiltonian $\mathbb{H}_{N}(-i \nabla)$ with a band index $N$ specifies the bulk electron states of NI, the two-spinor $\Phi_{N}(\mathbf{r})=\left(\phi_{N}^{\uparrow}(\mathbf{r}), \phi_{N}^{\downarrow}(\mathbf{r})\right)^{\operatorname{tr}}$ is represented in the Bloch basis functions $w_{N}=\{|N, \uparrow\rangle,|N, \downarrow\rangle\}$. We regard NI as a wide-gap semiconductor without SOC and presume that the TI bulk gap $2 \Xi$ lies entirely inside the NI bulk gap on the energy scale. The spinor components $\theta_{j}(\mathbf{r})$ (the subscript $j$ numbers the corresponding component; $j=1,2,3,4)$ and $\phi_{N}^{\uparrow \downarrow}(\mathbf{r})$ are treated as envelope functions in the frame of $\mathbf{k} \cdot \mathbf{p}$ method, i.e., they are smooth and continuous functions in the corresponding half spaces.

It was remarked above, that 3D TI and NI forming real TI/NI heterostructure are narrow-gap and wide-gap semiconductors, respectively, which posses different electron affinities. Therefore, drastic charge redistribution are thought to entail important electrostatic effects [7,31]. The presence of screening carriers, which can originate from the bound interfacial states (within our model, bulk-carrier-related screening can be readily ruled out), gives rise to a significant redistribution of the carrier density $n(\mathbf{r})$ within the subinterfacial region on the narrow-gap TI side. As a result, the band bending, which is proportional to a long-range part of the Coulomb potential in 
Eq. (3), $\varphi(\mathbf{r})=\int d \mathbf{r}^{\prime} \mathcal{V}\left(\mathbf{r}-\mathbf{r}^{\prime}\right) n\left(\mathbf{r}^{\prime}\right)$ [where $\mathcal{V}(\mathbf{r})$ is electronelectron interaction in the TI material, $\mathbb{I}$ is an unit $4 \times 4$ matrix], can remarkably contribute to the low-energy physics of the TI/NI heterostructures. It is obvious that significant charge redistribution takes place mostly on the narrow-gap TI side of the interface. While this effect, being weak on the wide-gap NI side, is neglected in Eq. (4).

For concrete TI/NI heterostructures, the self-consistent calculations of the potential $\varphi(\mathbf{r})$ [as well as the interaction $\mathcal{V}(\mathbf{r})]$ and the carrier density $n(\mathbf{r})$ may be carried out within the numerical ( $a b$ initio) approaches. To estimate the role of this potential analytically, one has to make some rough assumptions. First, the interaction $\mathcal{V}(\mathbf{r})$ is supposed to be independent of the orbital indices. Second, one averages the charge redistribution $n(\mathbf{r})$ over the $(x, y)$ plane remaining one-dimensional profile $n(z)$. This means that, on the TI side, electrons are implied to be subjected to the one-dimensional potential field $\varphi(z)$ smoothly varying (on the atomic scale) in the $z$ direction and homogeneous in the interface plane. Third, the scale of spatial variation of $\varphi(z)$ denoted as $l$ exceeds or is of the order of the Debye screening length in the bulk of the TI material. In the TI/NI heterostructures under consideration, the Coulomb effects are not small, so the magnitude of the potential $\varphi(z)$ may turn out to be larger than the TI bulk gap. Thus, the band bending could be large enough to modify remarkably the electron characteristics of the TI near the interface.

The $\mathbf{k} \cdot \mathbf{p}$ method cannot provide information on the wave-function behavior in the vicinity of the atomically sharp interface, where large momenta are highly important. So, the use of an envelope function method formally falls down in such the situation. To overcome this serious and well-known drawback (see discussion of a similar problem for a contact of two normal semiconductors in Ref. [4]), one should introduce the boundary conditions at the interface matching the envelope functions and their derivatives on the TI and NI sides. Following Ref. [33], one can formally write the boundary conditions in the matrix form with any model parameters. Unfortunately, it is not clear what set of parameters is more appropriate to describe the system under consideration, since the bulk wave functions of the TI and NI materials are classified according to different irreducible representations of different crystalline symmetry groups. So, one has no special receipt to minimize the number of components of the boundary-conditions matrix.

Below, according to Ref. [9], we use a simple variational approach and formally introduce an effective intermixing of electron states of the contacting TI and NI materials at the interface. The intermixing is simulated by an effective potential of hybridization $\mathbb{V}_{N}(\mathbf{r})$ between the states $| \pm, \uparrow \downarrow\rangle$ and $|N, \uparrow \downarrow\rangle$ specified by the eigen Bloch functions of the TI bulk and NI bulk, respectively, which are declared to be associated with different crystal symmetries. Really, the hybridization $\mathbb{V}_{N}(\mathbf{r})$ is nonzero in a small region $d$ (of the order a lattice parameter) around the geometrical boundary between the 3D TI and NI materials, where the applicability of the $\mathbf{k} \cdot \mathbf{p}$ scheme is violated. The length $d$ may be treated as an effective scale of the interface region. Introduction of the phenomenological term of the interface energy $\Omega_{I}$ enables us to correctly reconcile the long-range variations of the electron density on both sides of the interface at $|z|>d$ in the terms of the boundary conditions for the envelope functions $\Theta(\mathbf{r})$ and $\Phi_{N}(\mathbf{r})$. As long as the spatial variations of the sought interfacial states are sufficiently slow, one can adopt a local approximation for the interfacial potential; namely, $\mathbb{V}_{N}(\mathbf{r})=d \mathbb{V}_{N} \delta(z)$, where $\delta(z)$ is the $\delta$ function.

Because, in a plane geometry, the wave vector $\kappa=\left(k_{x}, k_{y}\right)$ is a good quantum number, one can deal with $\kappa$ harmonics of the envelope functions, $\Theta(\kappa, z)$ and $\Phi(\kappa, z)$, where $\Theta(\mathbf{r})=$ $\sum_{\kappa} \Theta(\boldsymbol{\kappa}, z) \exp (i \boldsymbol{\kappa} \boldsymbol{\rho}), \quad \Phi_{N}(\mathbf{r})=\sum_{\kappa} \Phi_{N}(\boldsymbol{\kappa}, z) \exp (i \boldsymbol{\kappa} \boldsymbol{\rho}), \boldsymbol{\rho}=$ $(x, y)$. Following the method described in Ref. [9] the equations for the envelope function on the TI side can be expressed in compact form as

$$
\begin{gathered}
{\left[\mathbb{H}_{T}(\boldsymbol{\kappa},-i \partial)+\varphi(z) \mathbb{I}-E \mathbb{I}\right] \Theta^{(N)}(\boldsymbol{\kappa}, z)=0,} \\
{\left.\left[\Pi(\boldsymbol{\kappa},-i \partial)-2 d \mathbb{U}^{(N)}(\boldsymbol{\kappa}, E)\right] \Theta^{(N)}(\boldsymbol{\kappa}, z)\right|_{z=0}=0 .}
\end{gathered}
$$

Here $E$ is the energy, $\Pi\left(\kappa, k_{z}\right)=i \partial \mathbb{H}_{T}(\mathbf{k}) / \partial k_{z}$ is the "current" density operator. We search for the solutions of Eqs. (6) and (7) that satisfy the condition $\Theta^{(N)}(\kappa, z \rightarrow \infty) \rightarrow 0$ when moving from the interface into the bulk. The localized states at the interface can appear only within the energy gap common for both the materials so that $|E(\kappa)|<\Xi<\left|E_{N}\right|$, where $E_{N}$ is the edge of the $N$ th band. The superscript $(N)$ indicates that the bound state with the envelope function $\Theta^{(N)}(\kappa, z)$ originates from the intermixing of the TI band state with the $N$ th state of the NI band. For a simplicity, in our model the direct tunneling of quantum states of the same symmetry from the one side of the interface to the other one is forbidden, while the tunneling (i.e., the hybridization $\mathbb{V}_{N}$ ) between states having different symmetries is permitted. Therefore, the boundary conditions of Eq. (7) involve an effective local pseudopotential $\mathbb{U}^{(N)}(\kappa, E)$ seen by electrons on the TI side. The magnitude of the $4 \times 4$ pseudopotential matrix elements can be written as $U_{j j^{\prime}}^{(N)}(\kappa, E)=d V_{j N} V_{N j^{\prime}}^{*} / h_{N}(\kappa, E)$, where $V_{j N}$ is the component of the matrix $\mathbb{V}_{N}$ specifying the intermixing between the $j$ th state of TI and the $N$ th state of $\mathrm{NI}, h_{N}(\kappa, E)$ is defined by the parameters of the $N$ th bulk band of NI and its position with respect to the TI bulk bands [9]. The parameters $U_{j j^{\prime}}^{(N)}$ characterize contributions of different chemical bonds to the TI/NI hybridization at the interface. For concreteness, we choose the situation corresponding to the plausible assumption that the $\mathrm{Bi}$ orbitals of the topmost quintuple layer (QL) of the $\mathrm{Bi}_{2} \mathrm{Se}_{3}(111)$ dominantly contribute to the chemical bond with the NI orbitals at the interface. In such the case the effective pseudopotential has four nonzero matrix elements: $U_{11}^{(N)}=U_{33}^{(N)}$ and $U_{13}^{(N)}=\left[U_{31}^{(N)}\right]^{*}$. It is worth noting that off-diagonal elements appear solely thanks to the spin-flip hybridization processes, which below are presumed to be negligible. We also omit the dependence of the interface potential on both $\kappa$ and $E$, which is quite correct upon condition that the ratio $\left|E_{N}\right| / \Xi$ is well larger than unity. Note, if $\left|E_{N}\right| \gg \Xi$, the estimation $h_{N} \sim \sqrt{\left|E_{N}\right| / m_{N}}$ is valid, where $m_{N}$ is an effective mass of the $N$ th band.

\section{MODIFICATION OF INTERFACIAL BOUND ELECTRON STATES UNDER BAND-BENDING EFFECT}

In the considered TI/NI heterostructure, the charge redistribution leads to arising of complicated and strongly inhomogeneous electrostatic field near the TI/NI boundary. 
Indeed, the positively (negatively) charged region of the thickness $\sim d$, in which chemical bonds between atomic layers adjacent to the TI/NI interface are rearranged, is screened by the electrons (holes) residing in the interfacial bound states and/or the band-bending-induced states of conduction (valence) bands. The spatial variation of the electrostatic potential $\varphi(z)$ is defined by the effective screening length $l \gg d$. Strictly speaking, the system of equations (6) and (7) for the envelope functions and the Poisson equation for $\varphi(z)$ have to be solved self-consistently, but it is evident that no exact analytical solution for such the task is available. Therefore, to capture the principal features of the band-bending effect on the evolution of interfacial electron states we shall use two distinct approximations, in which the $\varphi(z)$ is described either by a narrow-peak, when $l \ll \zeta$, or by a rectangular potential, when $l \gg \zeta$, where $\zeta$ is the envelope function scale.

In the case of a relatively long-range electrostatic potential, the condition $l \gg \zeta$ provides a possibility of the rather simple estimation for the band-bending effect on the spectrum of interfacial electron states by a simple shift of the energy scale in the TI part of the model Hamiltonian. Presuming that the probability density of the studied states is localized for the most part inside of the band-bending region, one can regard the electrostatic potential quasiclassically and approximate it roughly as the step function, $\varphi(z)=\varphi_{0}$ when $0<z<l$ and $\varphi(z)=0$ when $z>l$. Thus, in the near-interface region, the bound electron states are described by the equation $\left[\mathbb{H}_{T}(\kappa,-\right.$ $i \partial)-\bar{E} \mathbb{I}] \Theta^{(N)}(\boldsymbol{\kappa}, z)=0$ under the boundary condition (7), in which the energy is measured from the quantity $\varphi_{0}$, i.e., $E \rightarrow \bar{E}=E-\varphi_{0}$. This energy shift may be significant and even exceed half of the TI bulk gap, $\left|\varphi_{0}\right| \gtrsim \Xi$. Nevertheless, the quasiclassical approximation formally used here does not violate the applicability of the $\mathbf{k} \cdot \mathbf{p}$ method. Therefore, we can merely borrow the results of the work [9] taking into account the above-said energy shift $E \rightarrow \bar{E}=E-\varphi_{0}$ for the Dirac-like spectrum and characteristic lengths of the envelope functions. Strictly speaking, renormalized in this manner the interfacial bound states with a spectrum shifted by the band-bending effect may evolve to the quasiresonances due to their overlapping with the bulk states. Omitting a thorough discussion on the interfacial state features, it is worthy to emphasize that these features depend strongly on the effective pseudopotential $\mathbb{U}^{(N)}(\kappa, \bar{E})$ caused by the mixing between the TI and NI orbitals at the interface.

Now let us presume that a scale of change of $\varphi(z)$ is small as compared with the penetration depth of the interfacial electron bound state into the 3D TI bulk, i.e., $l \ll \zeta$. So, supposing that the relevant envelope function is situated principally outside of the band-bending region, to leading order in $l / \zeta$ we accept the utterly short-range form for the electrostatic potential, $\varphi(z)=$ $l \varphi_{0} \delta(z)$. Under this circumstance one can displace the potential $\varphi(z)$ from Eq. (6) into the boundary condition (7). Then the task on the interfacial bound states is reduced to the analytically solvable equation $\left[\mathbb{H}_{T}(\boldsymbol{\kappa},-i \partial)-E \mathbb{I}\right] \Theta^{(N)}(\boldsymbol{\kappa}, z)=0$ with the local interface potential renormalized as

$$
d \mathbb{U}^{(N)} \rightarrow d \mathbb{W}(N)=d \mathbb{U}^{(N)}+l \varphi_{0} \mathbb{I} .
$$

The effective interfacial potential in Eq. (8) is a $4 \times 4$ diagonal matrix $\mathbb{W}^{(N)}=\operatorname{diag}\left\{W_{1}^{(N)}, W_{2}^{(N)}, W_{3}^{(N)}, W_{4}^{(N)}\right\}$ with the fol- lowing components: $W_{2}^{(N)}=W_{4}^{(N)}=W_{2}=W_{4}=l \varphi_{0} / d$ and $W_{1}^{(N)}=W_{3}^{(N)}=W_{2}+U_{11}^{(N)}$. A comparison of these components allows us to discern two groups of states, whose behaviors clearly differ near the interface. The trial solution for each component of the envelope function spinor obeying the condition $\theta_{j}^{(N)}(\kappa, z \rightarrow \infty) \rightarrow 0$ can be represented as

$$
\begin{aligned}
\theta_{j}^{(N)}(\kappa, z)= & \alpha_{j}^{(N)}(\kappa, E) \exp \left[-q_{1}(\kappa, E) z\right] \\
& +\beta_{j}^{(N)}(\kappa, E) \exp \left[-q_{2}(\kappa, E) z\right],
\end{aligned}
$$

where the characteristic momenta

$$
\begin{gathered}
q_{1,2}(\kappa, E)=\sqrt{q_{1,2}^{2}(E)+\kappa^{2}}, \\
q_{1,2}^{2}(E)=\frac{\mathrm{A}^{2}-2 \mathrm{~B} \Xi \pm \sqrt{\mathrm{A}^{4}-4 \mathrm{~B} \Xi \mathrm{A}^{2}+4 \mathrm{~B}^{2} E^{2}}}{2 \mathrm{~B}^{2}}
\end{gathered}
$$

are the roots of the corresponding secular equation $\operatorname{det}\left[\mathbb{H}_{T}(\kappa, i q)-E \mathbb{I}\right]=0, \quad \kappa=|\kappa|, \quad q_{1,2}(E)>0$. The coefficients $\alpha_{j}^{(N)}(\kappa, E)$ and $\beta_{j}^{(N)}(\kappa, E)$ are determined by the boundary conditions at the interface.

After some algebra one can obtain the relations between the energy and the in-plane momentum for the bound states at the interface, $E(\kappa)$. In a nearest vicinity of the $\kappa=0$ point, the expression for the dispersion is described by the Dirac-conelike law $E(\kappa)=E(0) \pm v \kappa$, where $E(0)$ and $v$ are the node point position and the group velocity, respectively, which are functions of the interfacial potential $\mathbb{W}^{(N)}(8)$. The dependence of the Dirac point $E(0)$ on the interfacial potential components $W_{1,2}^{(N)}$ is given by the equation

$$
\begin{aligned}
0= & \mathrm{A}^{2} q_{1}(E) q_{2}(E)-\left[\mathrm{B} q_{1}^{2}(E)+\Xi-E\right] \\
& \times\left[\mathrm{B} q_{2}^{2}(E)+\Xi-E\right]+2\left\{q_{1}(E) q_{2}(E)-\frac{\Xi-E}{\mathrm{~B}}\right\} \\
& \times\left\{\mathrm{B}^{2} q_{1}(E) q_{2}(E)-d W_{2} d W_{1}^{(N)}-\frac{\mathrm{A}^{2}}{4}\right\} \\
& -2\left[q_{1}(E)+q_{2}(E)\right]\left[d W_{1}^{(N)}(\Xi-E)\right. \\
& \left.+d W_{2} \mathrm{~B} q_{1}(E) q_{2}(E)\right] .
\end{aligned}
$$

The results of numerical investigation of Eq. (12) are plotted in Figs. 1 and 2. The shaded areas in Fig. 1 denote the realm of existence of the interfacial bound states, that are the regions on the $\left(U_{11}^{(N)}, W_{2}\right)$ plane where $|E(0)|<\Xi$. In Fig. 2, the energy $E(0)$ is plotted with respect to $W_{2}$ for several values of $U_{11}^{(N)}$. We see rather the nonmonotonic behavior of the Dirac node point position vs the electrostatic potential, which in addition depends sensitively on the value and sign of the interface hybridization. With increasing the electrostatic potential amplitude, the node point evolves almost linearly from the origin, where $E(0)=0$ and $W_{2}=-U_{11}^{(N)} / 2$, until it merges into the continuum of the conduction (valence) bulk band, then it splits from the valence (conduction) bulk band and eventually approaches asymptotically the middle of the bulk gap, $E(0)=0 \rightarrow \pm 0$ when $W_{2} \rightarrow \mp \infty$. Looking at this plot, it is clear that the Dirac point position depends parametrically on the interface hybridization strength. In other words, given the embedded electrostatic potential, for $N$ labeling the NI 


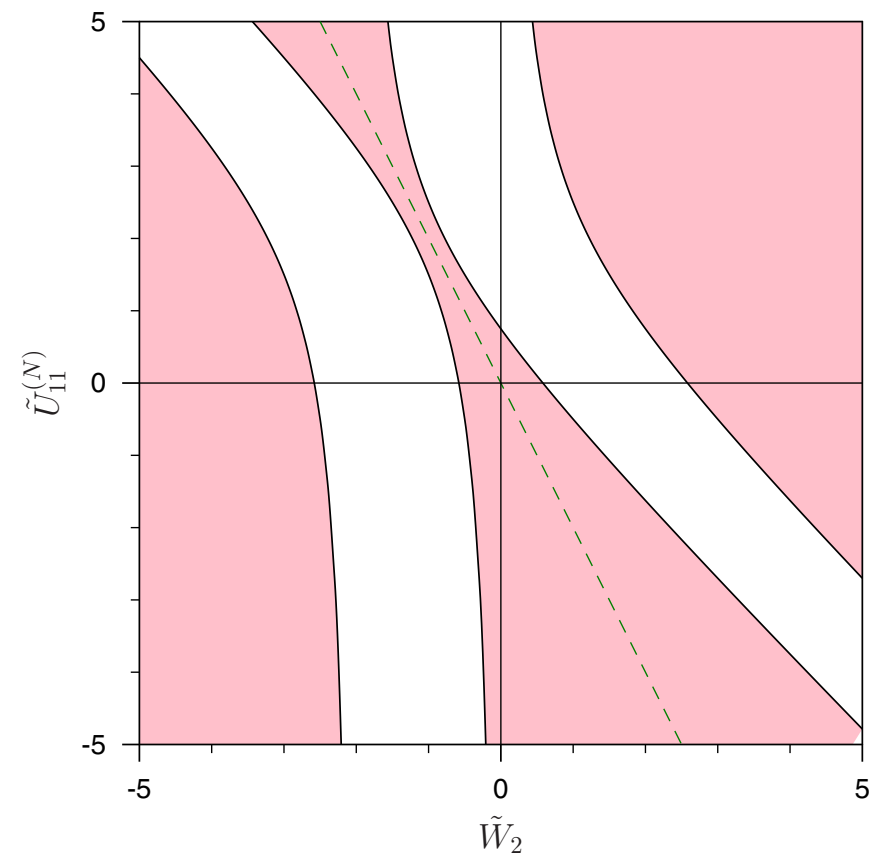

FIG. 1. (Color online) Realm of the interfacial bound state as a function of the embedded electrostatic potential $\widetilde{W}_{2}$ and the interfacial hybridization $\widetilde{U}_{11}^{(N)}$. In the dashed areas on the $\left(\widetilde{U}_{11}^{(N)}, \widetilde{W}_{2}\right)$ plane, the Dirac point lives inside the bulk band gap, $|\widetilde{E}(0)|<1$. The thick curves edging (bordering) the areas are defined by Eq. (12) under $\widetilde{E}(0)= \pm 1$, while the thin line is defined by Eq. (12) under $\widetilde{E}(0)=$ 0 . The dimensionless units are used: $\widetilde{U}_{11}^{(N)}=d U_{11}^{(N)} / \sqrt{\mathrm{B} \Xi}, \widetilde{W}_{2}=$ $d W_{2} / \sqrt{\mathrm{B} \Xi}, \widetilde{E}(0)=E(0) / \Xi$.

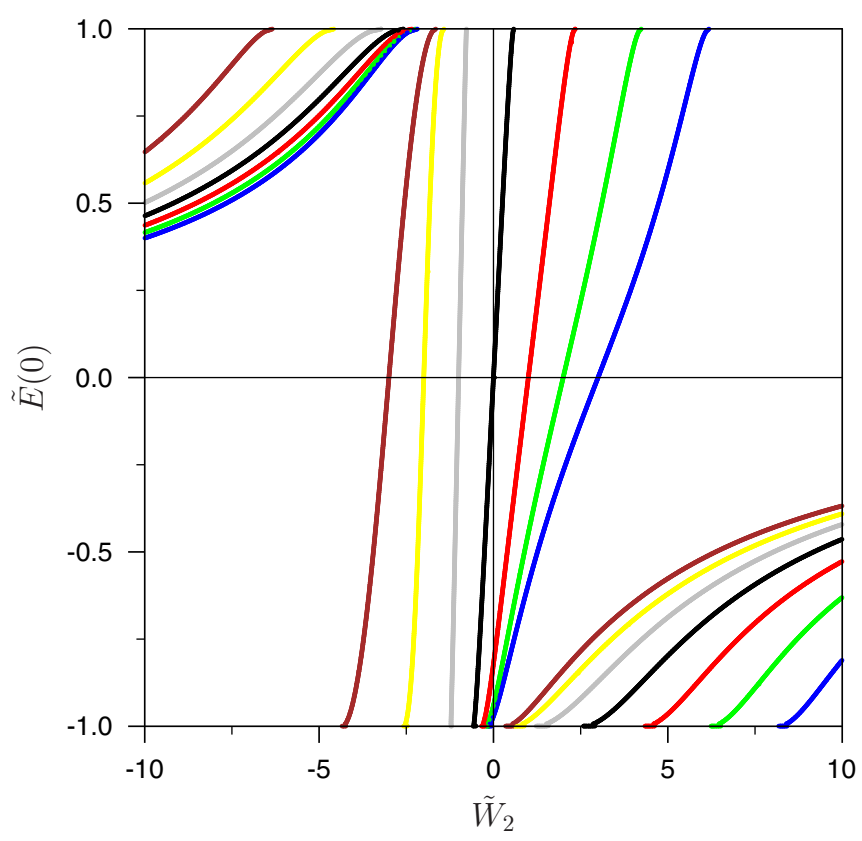

FIG. 2. (Color online) Position of the node point $\widetilde{E}(0)$ versus the electrostatic potential $\widetilde{W}_{2}$ for several values of the interface hybridization, $\widetilde{U}_{11}^{(N)}: \widetilde{U}_{11}^{(N)}=-6$ (blue), $\widetilde{U}_{11}^{(N)}=-4$ (green), $\widetilde{U}_{11}^{(N)}=$ -2 (red), $\widetilde{U}_{11}^{(N)}=0$ (black), $\widetilde{U}_{11}^{(N)}=2$ (gray), $\widetilde{U}_{11}^{(N)}=4$ (yellow), $\widetilde{U}_{11}^{(N)}=6$ (brown). The corresponding dependencies are plotted from left to right. Dimensionless units are used: $\widetilde{U}_{11}^{(N)}=d U_{11}^{(N)} / \sqrt{\mathrm{B} \Xi}$, $\widetilde{W}_{2}=d W_{2} / \sqrt{\mathrm{B} \Xi}, \lambda=\mathrm{A}^{2} / 4 \mathrm{~B} \Xi=1.5, \widetilde{E}(0)=E(0) / \Xi$. bulk band hybridized with the TI bulk bands (1), there exists the eigen bound state with the energy $E^{(N)}(0)$ lying inside the gap. Thereby, several hybridized states can appear at the interface. However, it is worth noting that sometime the energy level $E^{(N)}(0)$ can leave the gap merging into the bulk band continuum.

A more thorough picture of the envelope function evolution with potential strength is represented in Ref. [29].

One can describe analytically two extreme limits. Under the weak interface potential, $\left|W_{1,2}^{(N)}\right| \ll \sqrt{\mathrm{B} \Xi}$, the Dirac point shifts with respect to the middle of the gap, up or down depending on the sign of $W_{2}+\left|W_{1}^{(N)}\right|$ :

$$
E^{(N)}(\kappa)= \pm|\mathrm{A}| \kappa+\frac{4|\mathrm{~A}| \Xi(\kappa)}{\mathrm{A}^{2}+4 \mathrm{~B} \Xi(\kappa)}\left(2 d W_{2}+d U_{11}^{(N)}\right),
$$

where $\Xi(\kappa)=\Xi-\mathrm{B} \kappa^{2}$. Note that the group velocity near the node point does not change with $W_{1,2}^{(N)}$, but the spectrum deviates slightly from the linear dependence. In the limit $\left|W_{1,2}^{(N)}\right| \rightarrow 0$, the coordinate dependence of the envelope function is described as a sum of two exponents,

$$
\theta_{j}^{(N)}(\kappa, z) \sim \exp \left[-q_{1}(\kappa) z\right]+\exp \left[-q_{2}(\kappa) z\right],
$$

where the characteristic momenta are

$$
q_{1,2}^{0}(\kappa)=q_{1,2}(\kappa,|E|=|\mathrm{A}| \kappa)=\frac{|\mathrm{A}| \pm \sqrt{\mathrm{A}^{2}-4 \mathrm{~B} \Xi(\kappa)}}{2 \mathrm{~B}} .
$$

So, the probability density of the interfacial electron states is peaked on the boundary $z=0$ and its tail penetrates into the TI bulk with the decay length $\left(q_{2}^{0}\right)^{-1}$.

One can also describe analytically the opposite limit, $\left|W_{1,2}^{(N)}\right| \gg \mathrm{B} q_{1}^{0} \simeq|\mathrm{A}|$, when a very strong electrostatic potential provides the "hard-wall" boundary conditions at $z=0$. For $N=1$ labeling the corresponding interfacial bound state, the dispersion approaches the linear law as

$$
E^{(1)}(\kappa)= \pm|\mathrm{A}| \kappa-|\mathrm{A}| \Xi(\kappa)\left(\frac{1}{d W_{2}}+\frac{1}{d W_{2}+U_{11}^{(1)}}\right) .
$$

So, the spectrum of bound electron states acquires the nodepoint shift and curvature; however, the effective velocity $|\mathrm{A}|$ near the node point does not change since the amendment does not contain a term linear in the momentum $\kappa$. In the limit $\left|W_{1,2}^{(1)}\right| \rightarrow \infty$, the coordinate dependence of the envelope function is described as a difference of the exponents,

$$
\theta_{j}^{(1)}(\kappa, z) \sim \exp \left[-q_{1}(\kappa) z\right]-\exp \left[-q_{2}(\kappa) z\right],
$$

where the characteristic momenta are given by Eq. (15). The maximum of the electron density, $\left|\theta_{j}^{(1)}(z)\right|^{2}$, occurs near the point $z_{0}=\ln \left(q_{1}^{0} / q_{2}^{0}\right) /\left(q_{1}^{0}-q_{2}^{0}\right)<\sqrt{\mathrm{B} / \Xi}<\left(q_{2}^{0}\right)^{-1}$, which is distant from the interface.

Thus, we argue that, at $d<l \ll \zeta \simeq\left(q_{2}^{0}\right)^{-1}$, under the large value of an embedded electrostatic field, $\left|l \varphi_{0}\right| \gg \mathrm{B} q_{1}^{0}$, the TI/NI interface hosts the Dirac-like gapless state with the node point near the middle of the bulk gap, $E^{(1)}(\kappa=$ $0) \sim\left|W_{1,2}^{(1)}\right|^{-1} \rightarrow 0$, which displays low amplitude at the interface, $\theta_{j}^{(1)}(z=0) \sim\left|W_{1,2}^{(1)}\right|^{-2} \rightarrow 0$. Note the envelope function associated with the NI bulk band labeled as $N=1$ is 
negligibly small in the entire half space $z<0, \Phi_{1}(z) \sim \Theta^{(1)}$ $(z=0) \exp \left(\sqrt{2 m_{1}\left|E_{1}\right|} z\right)$ [31], due to the strong band bending such that $\left|l \varphi_{0}\right| \gg \mathrm{B} q_{1}^{0}$ and despite the finite intermixing at the interface. When $l \varphi_{0} \gg d U_{11}^{(1)}$, the latter inequality could be met thanks to the fact that the relevant NI band with $N=1$ is sufficiently distant from the TI bulk bands.

If the band-bending energy is not large, $\left|l \varphi_{0}\right| \simeq \mathrm{B} q_{1}^{0}$, the Dirac-like gapless state appears with the node point distant from the middle of the bulk gap, $E^{(2)}(\kappa=0) \lesssim \Xi$. The envelope function of this bound state indicated by $N=2$ has a finite amplitude on either the TI or NI side of the interface, at that $\Phi_{2}(z=0) \sim \Theta^{(2)}(z=0)$.

\section{NUMERICAL CALCULATION RESULTS}

By means of relativistic DFT calculations we studied the electronic band structure of $\mathrm{Bi}_{2} \mathrm{Se}_{3}$ film on $\mathrm{ZnSe}$ substrate.

For calculations we use the Vienna Ab Initio Simulation Package (VASP) $[34,35]$ with the generalized gradient approximation (GGA) [36] to the exchange correlation potential. The interaction between the ion cores and valence electrons was described by the projector augmented-wave method $[37,38]$. Relativistic effects, including SOC, were taken into account. The kinetic cutoff energy for the plane-wave basis set was $350 \mathrm{eV}$, and a $9 \times 9 \times 1 k$-point mesh was used to sample the $2 \mathrm{D}$ Brillouin zone (BZ). To simulate the (111) interfaces between $\mathrm{Bi}_{2} \mathrm{Se}_{3}$ film and zincblende $\mathrm{ZnSe}$ the lattice constants of the substrate in hexagonal (111) plane were fixed to that of $\mathrm{Bi}_{2} \mathrm{Se}_{3}$. The (111) hexagonal parameter of cubic $\mathrm{ZnSe}$ is $\approx 2 \%$ smaller then $\mathrm{Bi}_{2} \mathrm{Se}_{3}$ parameter. The optimization of the structure of $\mathrm{ZnSe}$ with the fixed parameter in (111) plane results in small $(<4 \%)$ contraction of interlayer spacings in the [111] direction. $\mathrm{The} \mathrm{Bi}_{2} \mathrm{Se}_{3}$ film was taken of five-QL thickness. The $\mathrm{ZnSe}$ substrate was modeled by a slab consisting of nine bilayers that is over $20 \AA$ in thickness. The most stable interfaces between $\mathrm{Bi}_{2} \mathrm{Se}_{3}$ film and the $\mathrm{ZnSe}$ were found for in-plane matching where the interface $\mathrm{Se}$ atom belongs to both $\mathrm{Bi}_{2} \mathrm{Se}_{3}$ and substrate sublattices [see Fig. 3(a)]. The atomic positions within the four near-interface atomic layers of $\mathrm{Bi}_{2} \mathrm{Se}_{3}$ thin film and the substrate were optimized. The geometry optimization is performed until the residual forces were smaller than $10 \mathrm{meV} / \AA$. A vacuum spacer of $\sim 20 \AA$ was included to ensure negligible interaction between opposite sides of the structure. The free surface of the $\mathrm{ZnSe}$ slab was passivated by hydrogen atoms.

The crystal structure of the zincblende $\mathrm{ZnSe}$ semiconductor can be described as the alternating sequence of anion and cation (111) layers (our calculation gives $\mathrm{Zn}^{+0.77}$ and $\mathrm{Se}^{-0.77}$ ). A direct consequence of this geometry is that the surface of substrate carries a finite charge. In the case of $\mathrm{Zn}$-terminated substrate considered here the charge is positive. As far as the $\mathrm{Bi}_{2} \mathrm{Se}_{3}$ is a covalent material and has no surface charge, the net charge at the interface should be positive, leading to a band bending at the interface.

We estimated the band-bending effect by calculating the difference between total electrostatic potential of $\mathrm{Bi}_{2} \mathrm{Se}_{3} / \mathrm{ZnSe}$ heterostructure and potentials of separated $\mathrm{ZnSe}$ and $\mathrm{Bi}_{2} \mathrm{Se}_{3}$ slabs. The resulting potential differences $\Delta V$ averaged over $x y$ planes for $\mathrm{ZnSe}(z<0)$ and $\mathrm{Bi}_{2} \mathrm{Se}_{3}(z>0)$ subsystems are shown in Fig. 3(b). As one can see, the moderate positive shift (a)

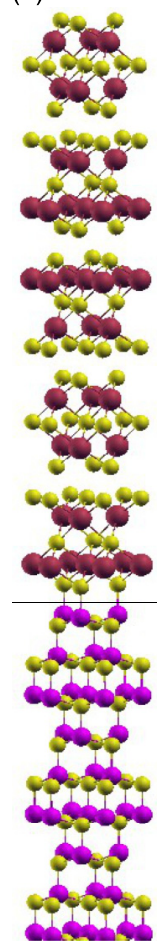

(b)

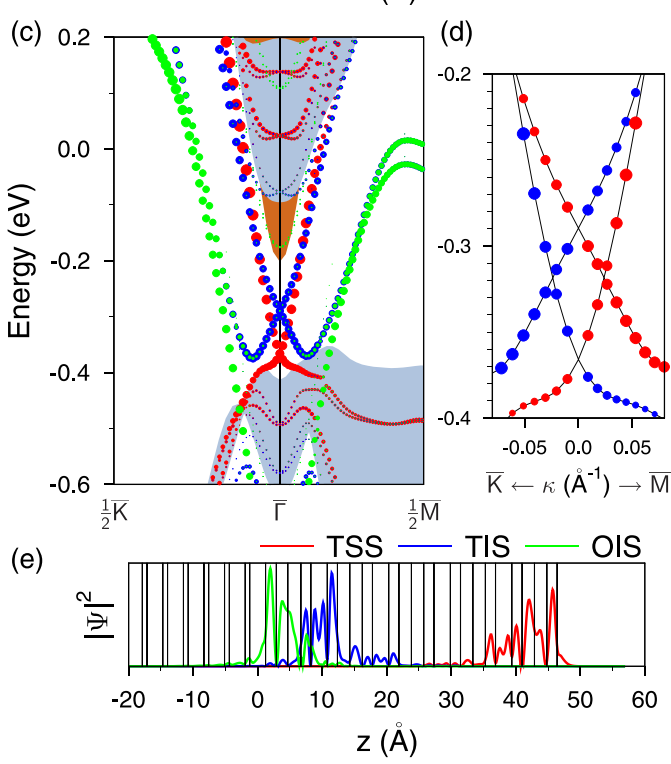

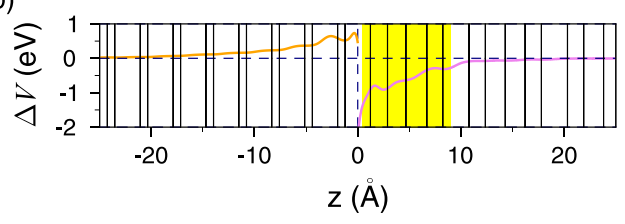

FIG. 3. (Color online) (a) Crystal structure of five-QL films of $\mathrm{Bi}_{2} \mathrm{Se}_{3}$ on the $\mathrm{ZnSe}$ substrate; maroon, yellow, and pink balls denote $\mathrm{Bi}, \mathrm{Se}$, and $\mathrm{Zn}$ atoms, respectively; horizontal line marks position of the interface plane. (b) Potential difference $\Delta V$ between total electrostatic potential of $\mathrm{Bi}_{2} \mathrm{Se}_{3} / \mathrm{ZnSe}$ heterostructure and potentials of $\mathrm{ZnSe}$ (orange line) and $\mathrm{Bi}_{2} \mathrm{Se}_{3}$ (violet line) slabs; vertical lines mark positions of the atomic layers; layers at negative $z$ are substrate layers; layers at positive $z$ are layers of $\mathrm{Bi}_{2} \mathrm{Se}_{3}$ film; $z=0$ correspond to interface plane; yellow rectangle marks first (interfacial) QL of $\mathrm{Bi}_{2} \mathrm{Se}_{3}$ film. (c) Electronic structure of $\mathrm{Bi}_{2} \mathrm{Se}_{3} / \mathrm{ZnSe}$; size of green circles corresponds to the weight of the states in the five atomic layers of $\mathrm{Bi}_{2} \mathrm{Se}_{3}$ closest to the interface; blue circles denote weight of the states in the next five layers of $\mathrm{Bi}_{2} \mathrm{Se}_{3}$; red circles are states localized at free surface of $\mathrm{Bi}_{2} \mathrm{Se}_{3}$ film; light steel blue and brown areas denote projections of the $\mathrm{Bi}_{2} \mathrm{Se}_{3}$ and $\mathrm{ZnSe}$ bulk states, respectively. (d) Spin polarization of the topological surface state (TSS) and topological interfacial state (TIS) where filled circles represent the value of the in-plane spin component (red and blue colors denote positive and negative values of spin, respectively). (e) Spatial localization of the ordinary interfacial state (OIS) (green line), TIS (blue line), and TSS (red line) in $\mathrm{Bi}_{2} \mathrm{Se}_{3} / \mathrm{ZnSe}$.

of the potential occurs in the $\mathrm{ZnSe}$ part of the heterostructure while the sizable negative potential difference was found within the interfacial (first) QL of the $\mathrm{Bi}_{2} \mathrm{Se}_{3}$ film [yellow stripe in Fig. 3(b)]. This potential bending should result in trapping of the electrons localized in the first QL and its shift to lower energies.

The electronic band structure of the $\mathrm{Bi}_{2} \mathrm{Se}_{3} / \mathrm{ZnSe}$ heterostructure is shown in Fig. 3(c). The spectrum demonstrates existence of two Dirac cones at the BZ center as well as the presence of ordinary bound states at large $\kappa$ in the same energy interval within the energy gap. The Dirac states presented in the supercell are the topological states resided at outer (free) 
and interfacial sides of the $\mathrm{Bi}_{2} \mathrm{Se}_{3}$ film. The Dirac point of the topological interfacial state (TIS) lies $\sim 70 \mathrm{meV}$ above the Dirac point of the vacuum-side topological surface state (TSS). At the same time, these states have opposite spin helicities [Fig. 3(d)] like in TSSs localized on the top and bottom surfaces of the free-standing TI slab. Note that both TSS and TIS have a tiny gap of $\sim 10^{-5} \mathrm{eV}$ at the $\bar{\Gamma}$ point that is below the energy resolution of DFT. These Dirac gaps are substantially smaller than DFT calculated gap (a few meV) in the Dirac state of the free-standing inversion-symmetric slab of five-QL thickness, where the Dirac topological states are coupled through the slab. The breaking of inversion symmetry in $\mathrm{Bi}_{2} \mathrm{Se}_{3}$ film owing to interface formation results in decoupling of the topological states and consequently leads to considerable decrease in the Dirac gap. The switch off the SOC in the calculation results in disappearing both $\bar{\Gamma}$ Dirac-like states whereas ordinary states at large $\kappa$ expanded within the band gap and trapped in the vicinity of the interface remain intact.

The spatial localization of the state resided on the vacuumside surface of $\mathrm{Bi}_{2} \mathrm{Se}_{3}$ in the heterostructure [Fig. 3(e), red line] is the same as compared with that in the free-standing $\mathrm{Bi}_{2} \mathrm{Se}_{3}$ slab [39]. In contrast, spatial localization of the state resided on the opposite side of the $\mathrm{Bi}_{2} \mathrm{Se}_{3}$ film, adjacent to $\mathrm{ZnSe}$ substrate, is modified [Fig. 3(e), blue line]. This state is relocated deep into the TI film, i.e., away from the interface plane, so that its probability maximum lies in the second QL. Thus, the TIS leaves the band-bending region. The similar behavior was found for the interfacial topological Dirac state in $\mathrm{Bi}_{2} \mathrm{Se}_{3} / \mathrm{MnSe}$ heterostructure [7]. The ordinary bound state in the $\mathrm{Bi}_{2} \mathrm{Se}_{3} / \mathrm{ZnSe}$ heterostructure [Fig. 3(e), green line] is mostly located within interfacial QL of $\mathrm{Bi}_{2} \mathrm{Se}_{3}$ with only a small tail extending within $\mathrm{ZnSe}$ bilayer, closest to the interface plane and thus differs from the spatial localization of the ordinary bound state found in $\mathrm{Bi}_{2} \mathrm{Se}_{3} / \mathrm{MnSe}$ heterostructure, where it propagates deep into the substrate layers. This difference is due to different atomic structure of the zincblende $\mathrm{ZnSe}(111)$ substrate having bilayer geometry.

\section{COMPARISON OF ANALYTICAL AND NUMERICAL RESULTS}

From the DFT calculations it is seen that two types of interfacial bound electron states coexist in the $\mathrm{Bi}_{2} \mathrm{Se}_{3} / \mathrm{ZnSe}$ system. The natural question arises of how this coexistence may be interpreted within the analytical model discussed in Secs. II and III. It is evident that this simple $\mathbf{k} \cdot \mathbf{p}$ scheme is unable to substitute for the numerical methods of Sec. IV, but the analytical approach provides a qualitative picture of the electronic band structure. The problem, however, is to correctly apply used approximations to the concrete situation.

It has been demonstrated [9] that parameters of interfacial bound electron states strongly depend on the effective local pseudopotential at the interface. Since this pseudopotential depends on the energy, it should be also redefined to take the band-bending effect into account following the approximations discussed in Secs. II and III. So the system of equations (6) and (7) for the envelope functions on the TI side of the interface may have not a unique solution, but rather several solutions corresponding to different self-consistent pseudopotentials, energy spectra, and parameters of interfacial electron bound states. To proceed with a comparison of the DFT numerical and $\mathbf{k} \cdot \mathbf{p}$ analytic results, we have to make some preliminary comments. First, from Sec. IV we see that electrostatic potential in the form of two asymmetric triangular teeth of the quantum-well or quantum-barrier type really appears at the $\mathrm{Bi}_{2} \mathrm{Se}_{3} / \mathrm{ZnSe}$ interface. On the $\mathrm{ZnSe}(\mathrm{NI})$ side, the barrier is relatively low $(0.5 \mathrm{eV})$ compared to the NI bulk band gap $(1.2 \mathrm{eV})$, while on the $\mathrm{Bi}_{2} \mathrm{Se}_{3}$ (TI) side, the well depth $(2 \mathrm{eV})$ significantly exceeds the TI bulk band gap $(0.3 \mathrm{eV})$. The barrier and well widths are 20 and $10 \AA$, respectively. In the model of Secs. II and III, we neglected for simplicity the bend bending on the NI side of the structure, due to a presumed large Debye screening length in the NI material as compared to the one in the TI material, which seems to be a good approximation. Thus, for $\mathrm{Bi}_{2} \mathrm{Se}_{3} / \mathrm{ZnSe}$ interface we can estimate the parameters of the electrostatic potential $\varphi(z)$ in Secs. II and III to be $l \approx 10 \AA, \varphi_{0} \approx 2 \mathrm{eV}$. Second, let us define the term "envelope-function scale" as applied to the situation under discussion. In Fig. 3(e), the density of probability function $|\Psi(z)|^{2}$ for either ordinary or topological interfacial state on the TI side has the form of the peak with the maximum at $z=Z_{p}$ and width $\xi$. It is natural to define the envelope-function scale as $\zeta=\max \left\{Z_{p}, \xi\right\}$ to characterize the approximation used for the band-bending effect.

In the model of Secs. II and III applied to the $\mathrm{Bi}_{2} \mathrm{Se}_{3} / \mathrm{ZnSe}$ system, the ordinary branch of the envelope function may be associated with the interfacial state, which is confined inside the well and pinned on the interface [green line in Fig. 3(e)]. This branch is described within the approximation of a large electrostatic potential shaped like a rectangle well of deepness $\varphi_{0} \gg \Xi$ and width $l \gg \zeta$. On the other hand, the topological branch of the envelope function is related to the interfacial state, which lies outside the well and is significantly relocated from the $\mathrm{Bi}_{2} \mathrm{Se}_{3} / \mathrm{ZnSe}$ interface [blue line in Fig. 3(e)]. This branch is described within the approximation of a large $\left(\varphi_{0} \gg \Xi\right)$ and narrow-peak $(l \ll \zeta)$ electrostatic potential. The approximation of a large potential is perfect for both states since $\varphi_{0} \approx 2 \mathrm{eV}, \Xi \approx 0.15 \mathrm{eV}$. Unfortunately, as far as the shape and scale of the potential $\varphi(z)$ are concerned, the situation is not as good. Indeed, from Fig. 3(e) one can estimate the envelope-function scales of the ordinary and topological interfacial states as $\zeta \approx \xi \approx 8 \AA$ and $\zeta \approx Z_{p} \approx 11 \AA$, respectively. It is understood that, although the potential scale of $l \approx 10 \AA$ falls into the actual interval $Z_{p}>l>\zeta$, both the long-range approximation $l \gg \zeta$ and the short-range approximation $l \ll Z_{p}$ are rough enough, so our analytical description of the ordinary and topological branches has only qualitative meaning.

Nevertheless, even within such the simple envelopefunction formalism one can capture the specific fingerprint of topology in the interface states. As we argued above, in Fig. 2, when the interface perturbation can be regarded as a delta peak, $l \ll \zeta$, the Dirac-point energy of the in-gap bound state evolves continuously as a function of the potential strength, $W_{2}<0$, until it merges with the continuum of the bulk valence band in order to appear then again being split off the conduction band and approach the zero level when $\left|\widetilde{W}_{2}\right| \rightarrow \infty$. Strictly speaking, the binding energy and envelope-function profile are related to the details of the pseudopotential matrix $\mathbb{U}^{(N)}(E)$. Furthermore, the interface induced charge 
redistribution generates electrostatic potential that varies drastically on nanometer scale in the vicinity of the TI/NI interface. The band-bending effect is analytically tractable within the rectangular-quantum-well approximation based on a small penetration depth of the bound state into TI as compared with the scale of changes of the electrostatic potential long-range component, $l \gg \zeta$. These assumptions allow us to predict that the TI/NI interface can host two interface states which behave differently near the interface (they have different exponents and scaling functions). In general, the band bending should be treated in a self-consistent manner. This task is completed via numerical calculations based on the DFT method. For the $\mathrm{Bi}_{2} \mathrm{Se}_{3} / \mathrm{ZnSe}$ heterostructure, we have found two interfacial states, OIS and TIS, which is in agreement with the analytical results.

\section{CONCLUSION}

In summary, basing on the results obtained, we describe the evolution of the interfacial bound electron states under the influence of charge redistribution and the corresponding band-bending effect near the TI/NI interface.

In the absence of both the interfacial potential and band bending, the bound state exists at the TI/NI interface due the bulk-boundary correspondence rule and its Dirac-like spectrum lies inside the bulk TI energy gap. The short-range interfacial potential shifts this spectrum to the top (bottom) of the gap, deforms the wave function of the interfacial bound state and can even transform it into the quasiresonance. However, it is impossible to distinctly determine the type of the interfacial state ("topological" or "ordinary") in this case.

If the band bending near the TI/NI interface is sufficiently small compared with the bulk TI energy gap, the situation does not drastically change irrespective of whether the electrostatic potential is a relatively long-range or a relatively short-range type on the scale of the interfacial bound-state localization. If the band bending exceeds the bulk TI energy gap, but the electrostatic potential has relatively long-range character, we also do not predict serious modifications in the character of the interfacial bound state. However, if the band bending exceeds the bulk TI energy gap and electrostatic potential has relatively short-range component, two bound states appear at the TI/NI interface.

In brief, in this work for a rather simple model of the TI/NI interface within the $\mathbf{k} \cdot \mathbf{p}$ scheme we analytically describe the spectrum of interfacial bound electron states. At certain conditions the spectrum has two branches: topological and ordinary which differ in the energy and spatial distribution. We have proved the existence of such states in the $\mathrm{Bi}_{2} \mathrm{Se}_{3} / \mathrm{ZnSe}$ system by means of DFT calculations.

Angle-resolved photoelectron spectroscopy (ARPES) and conductivity measurements in the $\mathrm{Bi}_{2} \mathrm{Te}_{3}$ thin films deposited on $\mathrm{Si}(111)$ [40,41], InP [42], GaAs [43], and $\mathrm{Al}_{2} \mathrm{O}_{3}$ [44] substrates, where good TI/NI interfaces are possible to fabricate, should be of great interest. The recent ARPES investigations of the $\mathrm{Bi}_{2} \mathrm{Se}_{3}$ thin films and $\mathrm{Bi}_{2} \mathrm{Te}_{3} / \mathrm{Bi}_{2} \mathrm{Se}_{3}$ heterostructures grown on a $\mathrm{Si}(111)$ substrate [23] clearly demonstrated that the band bending appearing in the TI film leads to substantial separation of the probability maxima and energy spectra of the surface (TSS) and interfacial (TIS and/or OIS) quasiparticles. Unfortunately, detailed study of the interfacial electron states in this system, which requires separation of TIS and OIS contributions, has not been performed yet. We believe that the unusual states considered above exist in different magnetic and nonmagnetic TI/NI heterostructures and can initiate intriguing physical phenomena.

\section{ACKNOWLEDGMENTS}

We acknowledge partial support from the Basque Country Government, Departamento de Educación, Universidades e Investigación (Grant No. IT-366-07), the Spanish Ministerio de Ciencia e Innovación (Grant No. FIS2010-19609-C02$00)$, the Ministry of Education and Science of Russian Federation (Grant No. 2.8575.2013), Russian Foundation for Basic Researches (Grants No. 13-02-00016 and No. 13-0212110-ofi-m), and Saint Petersburg State University (Project 11.50.202.2015). Numerical calculations were performed on the SKIF-Cyberia supercomputer at the National Research Tomsk State University.
[1] R. Yoshimi, A. Tsukazaki, K. Kikutake, J. G. Checkelsky, K. S. Takahashi, M. Kawasaki, and Y. Tokura, Nat. Mater. 13, 253 (2014).

[2] G. Wu, H. Chen, Y. Sun, X. Li, P. Cui, C. Franchini, J. Wang, X.-Q. Chen, and Z. Zhang, Sci. Rep. 3, 1233 (2013).

[3] X. Li, G. Zhang, G. Wu, H. Chen, D. Culcer, and Z. Zhang, Chin. Phys. B 22, 097306 (2013).

[4] E. L. Ivchenko and G. E. Pikus, Superlattices and Other Heterostructures (Symmetry and Optical Phenomena), Springer Series in Solid-State Sciences, edited by M. Cardona (SpringerVerlag, Berlin-Heidelberg, 1995); E. L. Ivchenko, Optical Spectroscopy of Semiconductor Nanostructures (Alpha Science, Harrow, 2005).

[5] M. Z. Hasan and C. L. Kane, Rev. Mod. Phys. 82, 3045 (2010).

[6] X. L. Qi and S. C. Zhang, Rev. Mod. Phys. 83, 1057 (2011).
[7] S. V.Eremeev, V. N. Men'shov, V. V. Tugushev, P. M. Echenique, and E. V. Chulkov, Phys. Rev. B 88, 144430 (2013).

[8] S. V. Eremeev, V. N. Men'shov, V. V. Tugushev, and E. V. Chulkov, Journal of Magnetism and Magnetic Materials, doi:10.1016/j.jmmm.2014.09.029.

[9] V. N. Men'shov, V. V. Tugushev, and E. V. Chulkov, JETP Lett. 97, 258 (2013).

[10] S. V. Eremeev, M. G. Vergniory, T. V. Menshchikova, A. A. Shaposhnikov, and E. V. Chulkov, New J. Phys. 14, 113030 (2012).

[11] M. G. Vergniory, T. V. Menshchikova, S. V. Eremeev, and E. V. Chulkov, JETP Lett. 95, 213 (2012).

[12] Y. Liu, Y. Y. Li, S. Rajput, D. Gilks, L. Lari, P. L. Galindo, M. Weinert, V. K. Lazarov, and L. Li, Nat. Phys. 10, 294 (2014).

[13] Y. Liu, Y. Y. Li, D. Gilks, V. K. Lazarov, M. Weinert, and L. Li, Phys. Rev. Lett. 110, 186804 (2013). 
[14] M. Bianchi, R. C. Hatch, J. Mi, B. B. Iversen, and P. Hofmann, Phys. Rev. Lett. 107, 086802 (2011).

[15] L. A. Wray, S. Xu, Y. Xia, A. V. Fedorov, D. Hsieh, H. Lin, A. Bansil, Y. S. Hor, R. J. Cava, and M. Z. Hasan, Nat. Phys. 7, 32 (2011).

[16] M. R. Scholz, J. Sánchez-Barriga, D. Marchenko, A. Varykhalov, A. Volykhov, L. V. Yashina, and O. Rader, Phys. Rev. Lett. 108, 256810 (2012).

[17] T. Valla, Z. H. Pan, D. Gardner, Y. S. Lee, and S. Chu, Phys. Rev. Lett. 108, 117601 (2012).

[18] S. V. Eremeev, G. Landolt, T. V. Menshchikova, B. Slomski, Y. M. Koroteev, Z. S. Aliev, M. B. Babanly, J. Henk, A. Ernst, L. Patthey, A. Eich, A. A. Khajetoorians, J. Hagemeister, O. Pietzsch, J. Wiebe, R. Wiesendanger, P. M. Echenique, S. S. Tsirkin, I. R. Amiraslanov, J. H. Dil, and E. V. Chulkov, Nat. Commun. 3, 635 (2012).

[19] L. Miao, Z. F. Wang, W. Ming, M. Y. Yao, M. Wang, F. Yang, Y. R. Song, F. Zhu, A. V. Fedorov, Z. Sun, C. L. Gao, C. Liu, Q. K. Xue, C. X. Liu, F. Liu, D. Q. and J. F. Jia, Proc. Natl. Acad. Sci. U. S. A. 110, 2758 (2013).

[20] T. V. Menshchikova, M. M. Otrokov, S. S. Tsirkin, D. A. Samorokov, V. V. Bebneva, A. Ernst, V. M. Kuznetsov, and E. V. Chulkov, Nano Lett. 13, 6064 (2013).

[21] Q. Zhang, Z. Zhang, Z. Zhu, U. Schwingenschlögl, and Y. Cui, ACS Nano 6, 2345 (2012).

[22] G. S. Jenkins, D. C. Schmadel, A. B. Sushkov, H. D. Drew, M. Bichler, G. Koblmueller, M. Brahlek, N. Bansal, and S. Oh, Phys. Rev. B 87, 155126 (2013).

[23] M. H. Berntsen, O. Götberg, B. M. Wojek, and O. Tjernberg, Phys. Rev. B 88, 195132 (2013).

[24] J. Chen, H. J. Qin, F. Yang, J. Liu, T. Guan, F. M. Qu, G. H. Zhang, J. R. Shi, X. C. Xie, C. L. Yang, K. H. Wu, Y. Q. Li, and L. Lu, Phys. Rev. Lett. 105, 176602 (2010).

[25] J. G. Checkelsky, Y. S. Hor, R. J. Cava, and N. P. Ong, Phys. Rev. Lett. 106, 196801 (2011).

[26] C. Chen, S. Hea, H. Wenga, W. Zhanga, L. Zhaoa, H. Liua, X. Jiaa, D. Moua, S. Liua, J. Hea, Y. Penga, Y. Fenga, Z. Xiea, G. Liua, X. Donga, J. Zhanga, X. Wangb, Q. Pengb, Z. Wangb, S. Zhangb, F. Yangb, C. Chenb, Z. Xub, X. Daia, Z. Fanga, and X. J. Zhou, Proc. Natl. Acad. Sci. U. S. A. 109, 3694 (2012).
[27] K. Park, C. De Beule, and B. Partoens, New J. Phys. 15, 113031 (2013).

[28] P. D. C. King, R. C. Hatch, M. Bianchi, R. Ovsyannikov, C. Lupulescu, G. Landolt, B. Slomski, J. H. Dil, D. Guan, J. L. Mi, E. D. L. Rienks, J. Fink, A. Lindblad, S. Svensson, S. Bao, G. Balakrishnan, B. B. Iversen, J. Osterwalder, W. Eberhardt, F. Baumberger, and Ph. Hofmann, Phys. Rev. Lett. 107, 096802 (2011).

[29] V. N. Menshov, V. V. Tugushev, T. V. Menshchikova, S. V. Eremeev, P. M. Echenique, and E. V. Chulkov, J. Phys.: Condens. Matter 26, 485003 (2014).

[30] K. Govaerts, K. Park, C. De Beule, B. Partoens, and D. Lamoen, Phys. Rev. B 90, 155124 (2014).

[31] V. N. Men'shov, V. V. Tugushev, S. V. Eremeev, P. M. Echenique, and E. V. Chulkov, Phys. Rev. B 88, 224401 (2013).

[32] C. X. Liu, X. L. Qi, H. J. Zhang, X. Dai, Z. Fang, and S. C. Zhang, Phys. Rev. B 82, 045122 (2010)

[33] V. V. Enaldiev, I. V. Zagorodnev, and V. A. Volkov, Pis'ma v ZhETF 101, 94 (2015).

[34] G. Kresse and J. Hafner, Phys. Rev. B 48, 13115 (1993).

[35] G. Kresse and J. Furthmüller, Comput. Mater. Sci. 6, 15 (1996).

[36] J. P. Perdew, K. Burke, and M. Ernzerhof, Phys. Rev. Lett. 77, 3865 (1996).

[37] P. E. Blöchl, Phys. Rev. B 50, 17953 (1994).

[38] G. Kresse and D. Joubert, Phys. Rev. B 59, 1758 (1999).

[39] S. V. Eremeev, Yu. M. Koroteev, E. V. Chulkov, JETP Lett. 91, 387 (2010).

[40] L. He, F. Xiu, Y. Wang, A. V. Fedorov, G. Huang, X. Kou, M. Lang, W. P. Beyermann, J. Zou, and K. L. Wang, J. Appl. Phys. 109, 103702 (2011).

[41] M. Vyshnepolsky, C. Klein, F. Klasing, A. Hanisch-Blicharski, and M. Horn-von Hoegen, Appl. Phys. Lett. 103, 111909 (2013).

[42] S. Schreyeck, N. V. Tarakina, G. Karczewski, C. Schumacher, T. Borzenko, C. Brüne, H. Buhmann, C. Gould, K. Brunner, and L. W. Molenkamp, Appl. Phys. Lett. 102, 041914 (2013).

[43] X. Liu, D. J. Smith, H. Cao, Y. P. Chen, J. Fan, Y.-H. Zhang, R. E. Pimpinella, M. Dobrowolska, and J. K. Furdyna, J. Vac. Sci. Technol. B 30, 02B103 (2012).

[44] P. Tabor, C. Keenan, S. Urazhdin, and D. Lederman, Appl. Phys. Lett. 99, 013111 (2011). 